University of Wollongong

Research Online

Faculty of Engineering and Information

Faculty of Engineering and Information

Sciences - Papers: Part A

Sciences

2012

\title{
A real-time in vivo dosimetric verification method for high-dose rate intracavitary brachytherapy of nasopharyngeal carcinoma
}

Zhen-Yu Qi

Sun Yat-Sen University, zyq210@uow.edu.au

Xiao-Wu Deng

Sun Yat-Sen University

$X$ Cao

Sun Yat-Sen University

Shao-Min Huang

Sun Yat-Sen University

Michael L. Lerch

University of Wollongong, mlerch@uow.edu.au

See next page for additional authors

Follow this and additional works at: https://ro.uow.edu.au/eispapers

Part of the Engineering Commons, and the Science and Technology Studies Commons

Research Online is the open access institutional repository for the University of Wollongong. For further information contact the UOW Library: research-pubs@uow.edu.au 


\title{
A real-time in vivo dosimetric verification method for high-dose rate intracavitary brachytherapy of nasopharyngeal carcinoma
}

\begin{abstract}
Purpose: A real-time in vivo dosimetric verification method using metal-oxide-semiconductor field effect transistor (MOSFET) dosimeters has been developed for patient dosimetry in high-dose rate (HDR) intracavitary brachytherapy of nasopharyngeal carcinoma (NPC). Methods: The necessary calibration and correction factors for MOSFET measurements in 192Iridium source were determined in a water phantom. With the detector placed inside a custom-made nasopharyngeal applicator, the actual dose delivered to the tumor was measured in vivo and compared to the calculated values using a commercial brachytherapy planning system. Results: Five MOSFETs were independently calibrated with the HDR source, yielding calibration factors of $0.48 \pm 0.007 \mathrm{cGymV}$. The maximum sensitivity variation was no more than 7 in the clinically relevant distance range of $1-5 \mathrm{~cm}$ from the source. A total of 70 in vivo measurements in 11 NPC patients demonstrated good agreement with the treatment planning. The mean differences between the planned and the actually delivered dose within a single treatment fraction were $-0.1 \pm 3.8$ and $-0.1 \pm 3.7$, respectively, for right and left side assessments. The maximum dose deviation was less than 8.5. Conclusions: In vivo measurement using the real-time MOSFET dosimetry system is possible to evaluate the actual dose to the tumor received by the patient during a treatment fraction and thus can offer another line of security to detect and prevent large errors.
\end{abstract}

\section{Keywords}

time, dosimetric, verification, method, high, dose, rate, intracavitary, brachytherapy, nasopharyngeal, carcinoma, real, vivo

\section{Disciplines}

Engineering | Science and Technology Studies

\section{Publication Details}

Qi, Z., Deng, X., Cao, X., Huang, S., Lerch, M. L. \& Rosenfeld, A. (2012). A real-time in vivo dosimetric verification method for high-dose rate intracavitary brachytherapy of nasopharyngeal carcinoma. Medical Physics, 39 (11), 6757-6763.

\section{Authors}

Zhen-Yu Qi, Xiao-Wu Deng, X Cao, Shao-Min Huang, Michael L. Lerch, and Anatoly B. Rosenfeld 


\title{
A real-time in vivo dosimetric verification method for high dose rate intracavitary brachytherapy of nasopharyngeal carcinoma
}

\author{
Zhen-Yu Qi, PhD, ${ }^{\mathrm{a}, \mathrm{b}} \quad$ Xiao-Wu Deng ${ }^{\mathrm{a}, \mathrm{b}}, \mathrm{PhD},{ }^{\mathrm{a}, \mathrm{b}} \quad$ Xin-ping Cao, $\mathrm{PhD}^{\mathrm{a}}$, \\ Shao-Min Huang, $\mathrm{PhD},{ }^{\mathrm{a}} \quad$ Michael Lerch, $\mathrm{PhD}^{\mathrm{b}}, \quad$ Anatoly Rosenfeld, $\mathrm{PhD}^{\mathrm{b}}$
}

${ }^{a}$ Department of Radiation Oncology, Sun Yat-Sen University Cancer Center and State Key Laboratory of Oncology in Southern China, Guangzhou 510060, China;

${ }^{\mathbf{b}}$ Centre for Medical Radiation Physics, University of Wollongong, Wollongong, NSW 2522, Australia

\section{Address correspondence to:}

Xiao-Wu Deng, $\mathrm{PhD}$

Department of Radiation Oncology

Sun Yat-Sen University Cancer Center

Guangzhou 510060, China.

Phone: (86) 20-87343036

Fax: (86) 20-87343044

Email: dengxw@mail.sysu.edu.cn

Running title: Real-time in vivo dosimetric verification for HDR brachytherapy 


\begin{abstract}
[Purpose]: A real-time in vivo dosimetric verification method using metal-oxide-semiconductor field effect transistor (MOSFET) dosimeters has been developed for patient dosimetry in high dose rate (HDR) intracavitary brachytherapy of nasopharyngeal carcinoma (NPC).
\end{abstract}

[Materials and Methods]: The necessary calibration and correction factors for MOSFET measurements in ${ }^{192}$ Iridium source were determined in a water phantom. With the detector placed inside a custom-made nasopharyngeal applicator, the actual dose delivered to the tumor was measured in vivo and compared to the calculated values using a commercial brachytherapy planning system (BPS).

[Results]: The mean calibration factor between each MOSFET detector in ${ }^{192}$ Iridium source was $0.48 \pm 0.007 \mathrm{cGy} / \mathrm{mV}$. The maximum sensitivity variation was no more than $7 \%$ in the clinically relevant distance range of 1 to $5 \mathrm{~cm}$ from the source. A total of 70 in vivo measurements in 11 NPC patients demonstrated good agreement with the treatment planning. The mean differences between the planned and the actually delivered dose within a single treatment fraction were $-0.1 \% \pm 3.8 \%$ and $-0.1 \% \pm 3.7 \%$, respectively, for right and left side assessments. Corresponding values obtained after averaging results over all fractions of a patient were $-0.2 \% \pm 3.9 \%$ (right side) and $-0.3 \% \pm 3.7 \%$ (left side). The maximum dose deviation was less than $8.5 \%$.

[Conclusion]: In vivo measurement using the real-time MOSFET dosimetry system is possible to evaluate the actual dose to the tumor received by the patient during a treatment fraction and thus can offer another line of security to detect and prevent large errors.

KEYWORDS: MOSFET, in vivo dosimetry, real time dosimetry, intracaviatry brachytehrapy, quality assurance 


\section{Introduction}

The recent evolution of computed tomography (CT)-based implant planning and dosimetry has sparked renewed interest in using high-dose rate (HDR) intracavitary brachytherapy for the treatment of nasopharyngeal carcinoma (NPC) [1, 2]. The CT-based techniques have accurately reconstructed the position of all intracavitary applicators with respect to the patient's anatomy, thereby demonstrating the spatial dose distributions to the tumor volume and neighboring structures. By optimizing the relative dwell times for each activated dwell position, the radiation doses are best conformed to the NPC whilst sparing more of the normal tissues like brain stem, spiral cord, temporary lobes, visual apparatus and pituitary gland [3].

However, when HDR brachytherapy is used, the treatments must be executed very carefully. A number of factors could potentially lead to substantial dose deviations from the prescription in the chain of HDR treatment [4]. This is mainly due to the fact that the planning and treatment systems tend to be relatively complicated. Significant errors in treatment dose can result from the use of incorrect calculation parameters as well as from machine malfunction [4-7]. As it has been pointed out recently [5], these potential errors are quite difficult to be identified and even though all the quality assurance (QA) checks recommended have been implemented, mistakes can happen without detection. This may result in severe radiation injury to patients since HDR brachytherapy delivers very high doses within a short time period. To avoid therapy misadministration as well as to verify the actual doses delivered to the tumor during 
an HDR treatment, the development of an in vivo dosimetry method as a supplement to existing QA programs is thus very desirable.

Several studies have explored the feasibility of in vivo dosimetry with thermoluminescent dosimeters (TLDs) and semiconductor diodes for HDR brachytherapy [5, 8-10]. While TLDs are often used in clinical dosimetry due to their small size, they are also known to be tedious to use $[11,12]$. The fact that acquiring moderately accurate, reproducible results using TLDs requires stringent calibration, handling and annealing procedures suggests that in vivo thermoluminescence dosimetry would be difficult to implement on a daily basis. Also TLDs are not real time dosimeters so that they are incompetent to provide on-line monitoring of operational mistakes and their immediate corrections. Semiconductor diodes are simpler to use than TLDs. The major disadvantages of diodes are their relatively large size, which makes them unable to be held in a small nasopharyngeal applicator. In addition, some investigations have revealed that diode measurements may need several correction factors for even simple application in clinical radiotherapy [13, 14].

The MOSFET detector is the most recent development for in vivo dosimetry compared with TLDs and diodes. Incorporating many of the advantages of TLDs and diodes such as small physical size, ease of use and immediate readout, the MOSFET has demonstrated their potential use in HDR brachytherapy [15-17]. In a previously published study, Zilio and his coworkers proved that the MOSFET detector, with appropriate calibration and correction, could also be used for absolute dosimetry in 
the context of a microSelectron HDR ${ }^{192}$ Ir source, even for situations where photon energy spectra were different from the reference location [15]. More recently, our group has successfully exploited an improved micro-MOSFET design with reduced external dimensions for use in brachytherapy catheters as a means of routine QA to verify brachytherapy dose calculations [16]. These pilot MOSFET dosimetry studies, basically, are limited to the integral dose measurements, in which results are obtained only after a treatment has been delivered. As reported by Gladstone et al [18] and others [19, 20], the MOSFET detector has an advantage over many other dosimeters in its ability to provide real-time dose information. This will offer extra benefits for treatment monitoring in that it may allow dose errors to be detected at a much earlier stage.

The main purpose of the present study is to provide a real time MOSFET dosimetry system in avoiding therapy misadministration for HDR intracavitary brachytherapy. The reproducibility of MOSFET measurements in real time mode was investigated. The preliminary results of real-time in vivo measurements with MOSFET detectors for 70 treatment fractions in 11 NPC patients are presented.

\section{Materials and Methods}

\section{Nasopharyngeal applicator}

HDR intracavitary brachytherapy treatments were generally delivered with two customized nasopharyngeal applicators at our institutions, one in each naris, with a 
separation of 1.2-1.5 cm according to the size of the tumor target.

The nasopharyngeal applicator (Figure 1) is a thermoplastic resin tube with a curvature of about $20^{\circ}$ at $1 \mathrm{~cm}$ from the distal blind end. It has an outer diameter of $2.0 \mathrm{~mm}$ and an inner diameter of $1.8 \mathrm{~mm}$, which can accommodate standard 6 French afterloading catheters. The applicator can be reshaped for each individual patient, when heated, to conform to the superoposterior wall of the nasopharynx. Thus the HDR ${ }^{192}$ Iridium stepping source can be positioned closer to the target mucosa as compared with the traditional intraluminal stabilizing devices which place the source in the middle of the cavity and hence up to $1-2 \mathrm{~cm}$ from the target mucosa [3].

The customization process for the nasopharyngeal applicator is performed under topical anesthesia using nebulized $4 \%$ lidocaine hydrochloride applied to the patient's nasal cavity, nasopharynx, and oropharynx. With the guidance of a nasopharyngeal speculum, the applicator is introduced

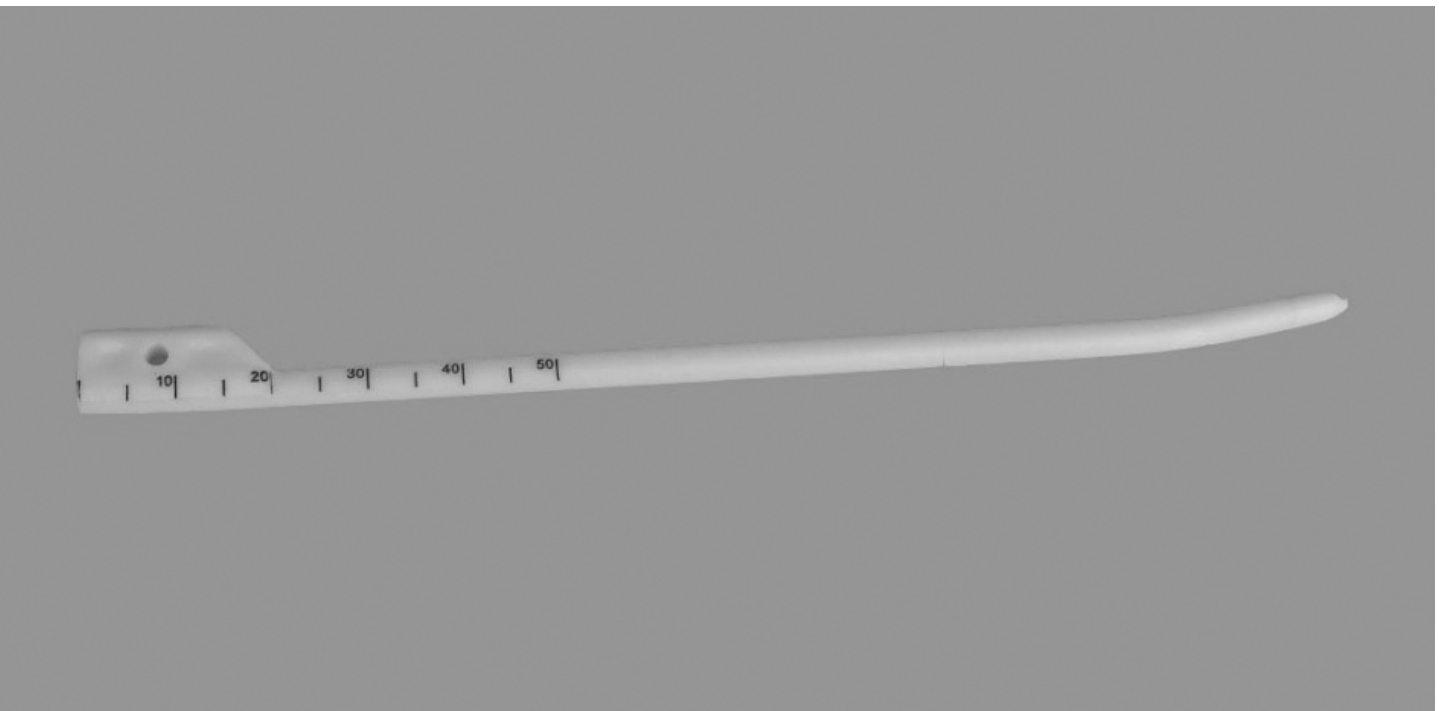


Figure 1 A custom-made nasopharyngeal applicator. The imprinted lengths and angles on the applicator are used for daily applicator positioning.

transnasally into the nasopharynx and is custom formed to be optimally positioned against the region of the primary disease. The nasopharyngeal applicator is then secured to the nose by a thermoplastic mask. The inserted length and orientation to the nasopharyngeal cavity are recorded accordingly from graduations and angles imprinted on the applicator to allow the applicator position to be reproduced in the subsequent treatments.

\section{MOSFET dosimetry system}

The applied MOSFET dosimetry system, including the recently developed micro-MOSFET detectors $\left(\mathrm{MOSkin}^{\mathrm{TM}}\right)$ and a microprocessor based reader, has been previously introduced $[16,21]$. One of the major characteristics of this micro-MOSFET detector is its packaging technology, by which the sensor chip is hermetically sealed with a $70-\mu \mathrm{m}$ thick water-equivalent film. The thin-film encapsulating layer prevents damage to the electronics, and acts as a carrier of the thin aluminum contact leads that are connected to the MOSFET sensor from the top-side.

In this experiment, dose measurements were performed in a real time mode with 
a positive bias voltage of $5 \mathrm{~V}$ applied to the MOSFET during irradiation. This required a connection to a laptop computer and utilization of the "MosPlot" software. The computer data acquisition system measured periodically the instantaneous voltage signal with the user-defined period (one second in this case). The software MosPlot 4.2 allowed for the online graphical presentation of the change in the threshold voltage $V_{\text {th }}$ (i.e. accumulated dose variation) or increments of $\Delta V_{\text {th }}$ for consecutive readouts (i.e. differential dose rate).

\section{MOSFET calibration and correction}

The calibration process is essentially a two-step process: calibration in a standard set-up to determine the reference calibration factor and subsequently the establishment of a series of correction factors for irradiation conditions deviating from the reference conditions.

Five MOSFET detectors were independently calibrated against a NE-2571 0.6 $\mathrm{cm}^{3}$ Farmer-type ionization chamber (Nuclear Technology Ltd., Reading, UK) in a 30 $\times 30 \times 30 \mathrm{~cm}^{3}$ water phantom. Following the recommendations for ionization dosimetry around the ${ }^{192}$ Iridium source, a reference point at a perpendicular distance of $5 \mathrm{~cm}$ from the source was selected [22]. By using a template calibration jig, the dose in water at the reference point was measured with the ionization chamber and the MOSFET detector, respectively. To improve the measurement accuracy, a radiation dose of 50 cGy was delivered to the MOSFET detector every time, which was sufficient to ensure that the response uncertainty associated with the MOSFET reader 
would equate to less than $1 \%$ of the threshold voltage change. The calibration factor, in terms of cGy per $\mathrm{mV}$, was therefore determined for each individual MOSFET.

To overcome the MOSFET sensitivity variation with the energy spectrum in

${ }^{192}$ Iridium brachytherapy, an energy dependent correction factor, CF, was introduced for measurement performed at locations other than the calibration position. The CFs, defined as the inverse of MOSFET sensitivities, were measured at five source-to-detector distances ranging from $1 \mathrm{~cm}$ to $5 \mathrm{~cm}$ with $1 \mathrm{~cm}$ increment along the source transverse bisector axis in the water phantom [16]. The derived CF factors were fitted with a least-squares polynomial, whereby the CFs for other positions between $1 \mathrm{~cm}$ and $5 \mathrm{~cm}$ could be obtained by interpolation. The determined energy dependent correction factors were compared with the published data for a previously used RADFET dosimeter.

\section{The reproducibility of real-time MOSFET dosimetry system}

Since the introduction of the automatic data acquisition system in the real time mode may add electronic noise to the signal, the readout reproducibility of the MOSFET dosimetry system in the real time mode was investigated at different dose levels and was compared with dosimeter readings being manually triggered.

Under the calibration set-up, the MOSFET detector was irradiated ten times for each of the following integrated doses: 20, 30, and $50 \mathrm{cGy}$. The threshold voltage change before and after each irradiation was measured using both the real-time mode 
and the manual mode. The corresponding coefficients of dispersion of repeated measurements were computed for different applied doses.

\section{CT-based HDR intracavitary brachytherapy}

HDR intracavitary brachytherapy provided an attractive method of boosting dose to NPC whilst sparing sensitive normal tissues. Prior to the CT simulation, the nasopharyngeal applicators were brought into the nasopharynx under the direct guidance of the nasopharyngeal speculum and remained in situ for the duration of the treatment. The patient was then laid on the simulator table, immobilized with a head and neck mask in the supine position. Three radiopaque markers were attached to the mask in alignment with the CT lasers, which served as benchmarks for daily radiographs to verify the applicator localization. After insertion of standard afterloading catheters with dummy sources into the nasopharyngeal applicator, the patient was CT scanned with 1-mm slice thickness and 1-mm pitch. The acquired CT images were subsequently transferred to the Plato BPS (Nucletron, The Netherlands).

The primary tumor target and critical structures such as brainstem, spinal cord and bilateral parotid glands were contoured on all the involved CT slices. The two applicators were sequentially reconstructed by the BPS. A catheter offset value, which was predetermined in the CT scout view, was used for correctly placing the center of the first dwell position with respect to the reconstructed catheter. The minimal peripheral dose (MPD), typically 3-5 Gy per fraction, was prescribed to cover the target surface. A geometrical optimization was performed, followed by manually 
adjusting dwell weights and times to further improve the target dose uniformity.

The dose rate that every source dwell position delivered to the measurement point where the MOSFET detector was located during the in vivo measurement was computed. This can be simply done by nulling all dwell times except for the selected dwell position. The real time accumulated dose (D) at any treatment time (t) was thus predicted using:

$$
D=\sum_{i=1}^{N} \int_{t_{i-1}}^{t_{i}} \dot{D}_{i} \cdot d_{t}
$$

where $\dot{D}_{i}$ is the dose rate delivered by source dwell position $i$ to the measurement point; $t_{i}$ is the planned dwell times of source dwell position $i$ and $t_{0}=0$.

\section{In vivo dosimetric verification}

The HDR treatments were delivered on an outpatient basis once per day for 2 to 4 days. The applicator localization was verified with fluoroscopy each time and orthogonal radiographs were taken for document.

The MOSFET detector was placed into one applicator fixing to its distal blind end, when the other applicator was being used to implement treatment and vice versa. Fixing the MOSFET to the distal blind end of the applicator may make the detector position reproducible with each fraction to the applicator.

After accurate positioning of the nasopharyngeal applicators and the MOSFET detector had been confirmed, the HDR treatment was performed sequentially for each 
applicator. The in vivo measurements were started as soon as the ${ }^{192}$ Iridium stepping source was driven out of the container. The accumulated threshold voltage of the MOSFET detector was recorded with the controlled software MosPlot 4.2 every second. By using a lookup table, the measured threshold voltage shift was converted to the absorbed dose, which was then compared with the calculated values by the BPS.

To date, real-time in vivo dosimetry with MOSFET detectors has been performed for a total of 70 measurements of 11 NPC patients. For all the patients, measurements were followed through the whole treatment sessions.

\section{Results}

\section{MOSFET calibration and correction}

Five MOSFET detectors were independently calibrated in the water phantom, resulting in a mean dose calibration factor of $0.46 \pm 0.012 \mathrm{cGy} / \mathrm{mV}$ for the ${ }^{192}$ Iridium source.

The energy dependent correction factors between the new micro-MOSFET detector and a previously used RADFET dosimeter provided by the same manufacturer were compared in Figure 2. The correction factors were plotted against the 


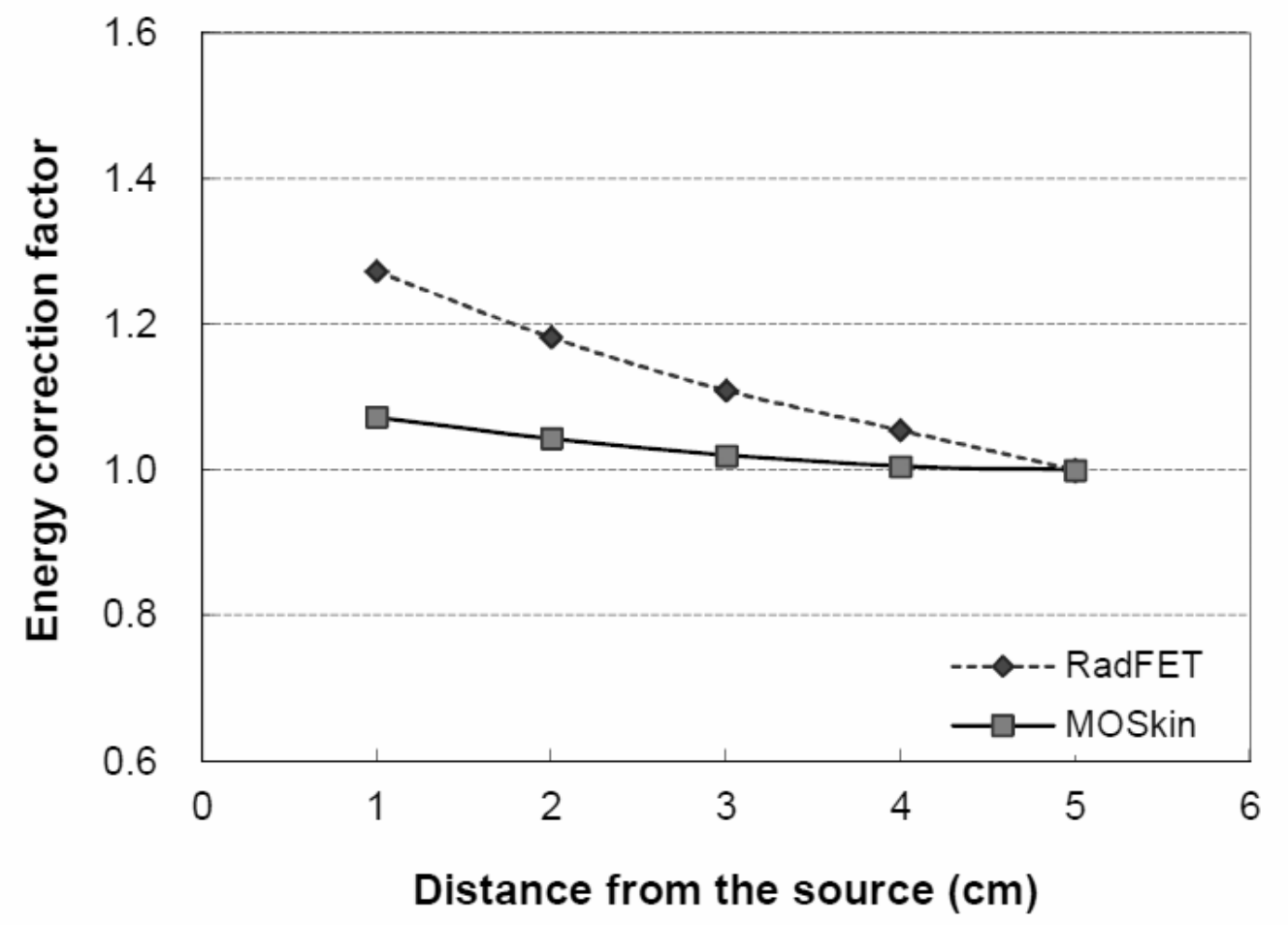

Figure 2 Comparison of energy-dependent correction factors between the new $\operatorname{MOSkin}^{\mathrm{TM}}$ and a previously used RADFET dosimeter. The RADFET data were derived from the published data [8], which were re-normalized to the 5-cm value.

source axis distances. The RADFET data were derived from the published data [15], which were re-normalized to the 5-cm value. The newly developed micro-MOSFET detector showed a relatively flat energy response in the low energy spectrum of the ${ }^{192}$ Iridium source compared with the RADFET dosimeter. The maximum sensitivity variation was found to be no more than $7 \%$ in the given distance range. 
As the radiation dose increased from $20 \mathrm{cGy}$ to $50 \mathrm{cGy}$, the reproducibility of the dosimetry system was improved from $3.5 \%$ to $1.4 \%$ for the manual mode and from $4.8 \%$ to $1.9 \%$ for the real-time mode (Table 1 ). A relatively large deviation in reproducibility tests associated with real-time data acquisition may be partially owing to electronic noise. However, for irradiated dose levels of more than $50 \mathrm{cGy}$, the real-time mode can also give a reasonable reproducibility of measurements better than $2.0 \%$.

\section{In vivo dosimetric verification}

In vivo dosimetric verification was performed for 70 measurements of 11 NPC patients. The residual position error of the nasopharyngeal applicators, observed from the verification films, was on average $1.6 \pm 0.6 \mathrm{~mm}$ in the right-left direction, $0.7 \pm 0.3 \mathrm{~mm}$ in the cranial-caudal direction, and $0.8 \pm 0.3 \mathrm{~mm}$ in the anterior-posterior direction.

Figure 3 was an example of real-time comparison of in vivo measurements with calculated accumulated dose variations for a given treatment fraction. The transit doses were included in the measurements. In low dose region $(<20 \mathrm{cGy})$, the maximum dose discrepancy between in vivo measurements and TPS calculations was less than 2 cGy. The MOSFET measurements coincided with the calculated values better than $\pm 3.5 \%$ in high dose region ( $>30 \mathrm{cGy}$ ). The measured total accumulated dose was found to be agreed with the planned dose within $1.1 \%$ in this case. 
The observed dose differences within a single treatment fraction were on average $-0.1 \% \pm 3.8 \%$ (right side) and $-0.1 \% \pm 3.7 \%$ (left side), as shown in Figure 4a and $4 \mathrm{~b}$. $94 \%$ of right side measurements and $91 \%$ of left side

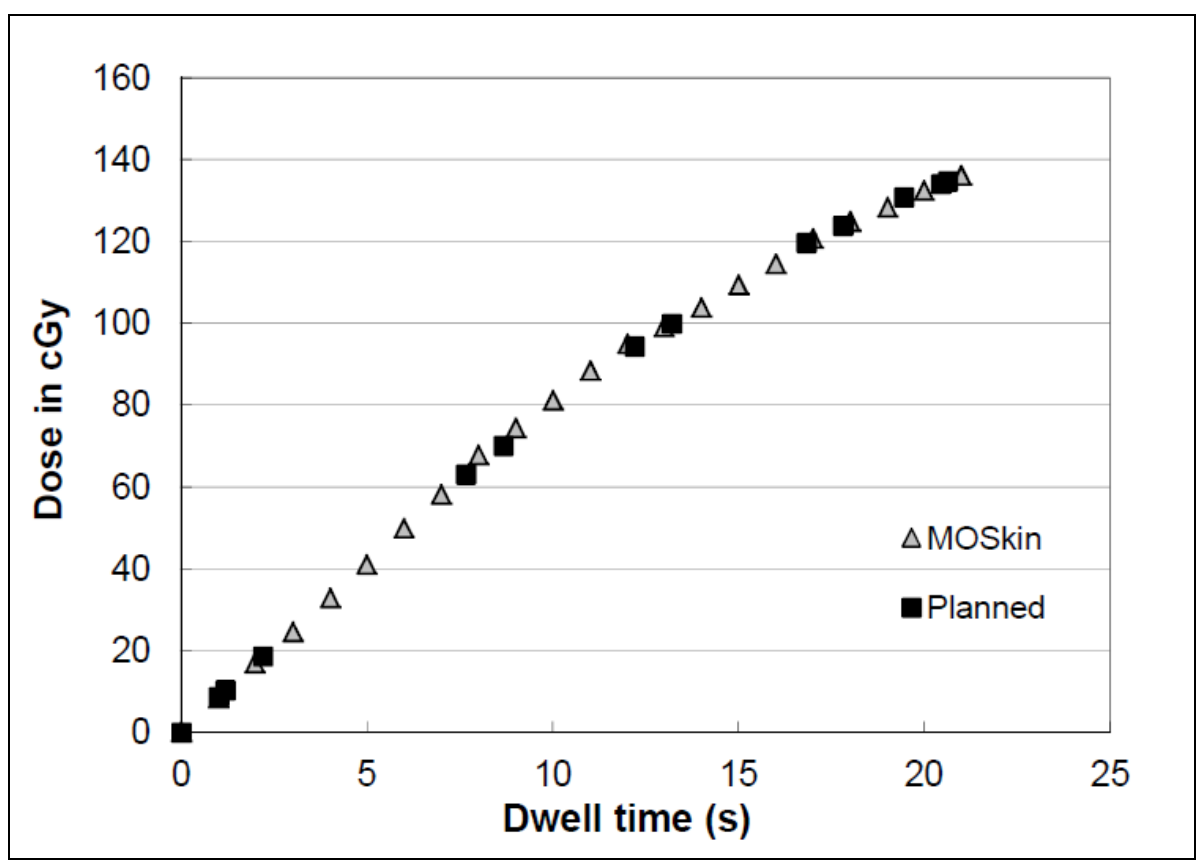

Figure 3 An example of real time dose variations between in vivo measurements and calculated accumulated doses for a given treatment fraction. The MOSFET measurements coincided with the calculated values throughout the treatment delivery. 

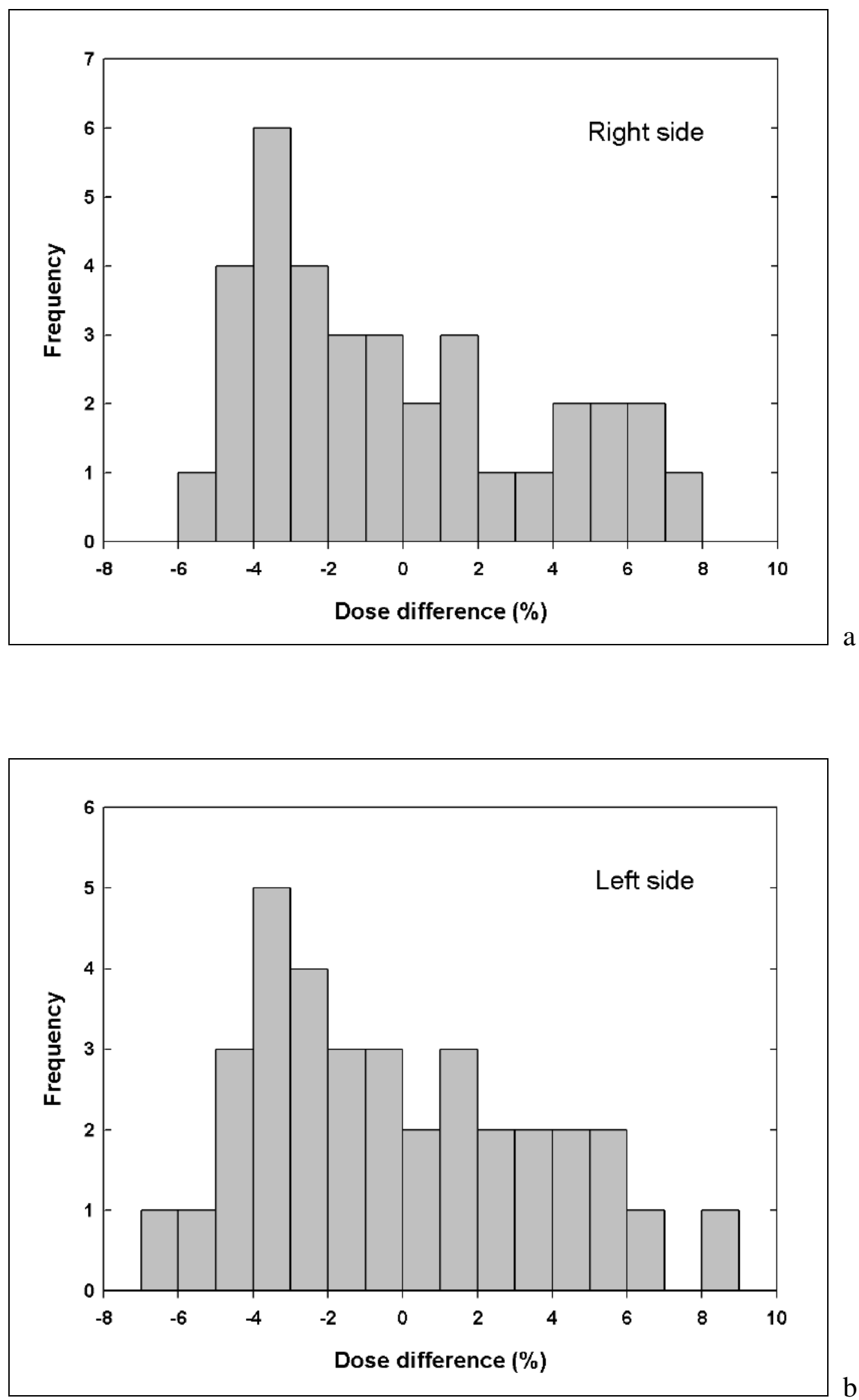

Figure 4 The frequencies of measurements with discrepancies in percentage between measured and calculated values for right and left side measurements, respectively. a: right side; b: left side. 


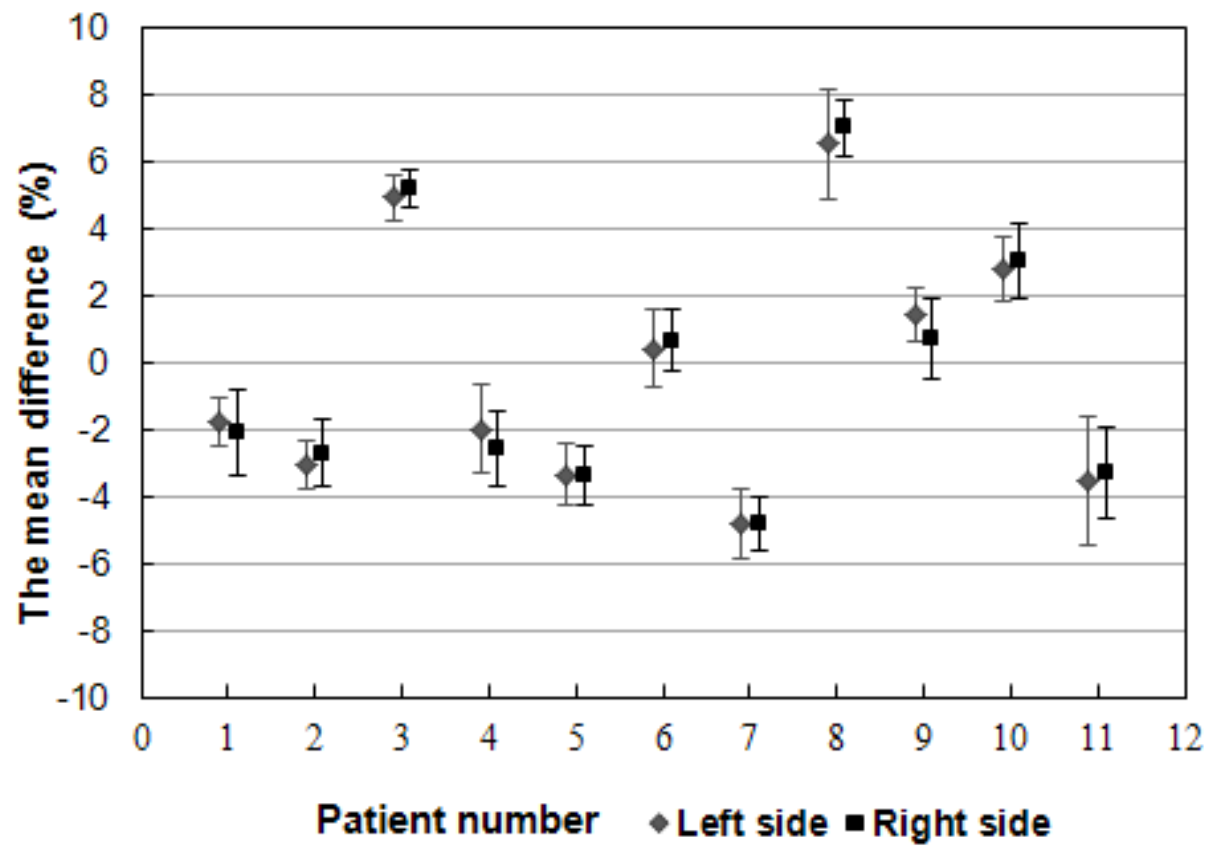

Figure 5 The discrepancies between measured and planned doses averaged over all fractions of a patient. The error bar represents one standard deviation.

assessments have deviations within the range of $-5 \%$ to $7 \%$. The maximum dose discrepancy was $8.3 \%$.

The corresponding dose measurements with MOSFET detectors in each fraction of a particular patient were then averaged and compared with the expected BPS calculated dose. As plotted in Figure 5, the mean discrepancies between measured and planned doses averaged over all fractions of each patient was $-0.2 \pm 3.9 \%$ (right side) and $-0.3 \pm 3.7 \%$ (left side), respectively. All the patients received a total dose different from the prescription within the tolerance range of $-5 \%$ to $7 \%$. 


\subsection{Discussions}

The widespread application of HDR brachytherapy with its inherent dangers necessitates a comprehensive set of QA programs to minimize risks to both patients and medical staff. Besides separate QA checks for each component of the treatment process, in vivo dosimetry which can provide an estimate of the overall error in an HDR treatment has been recommended by many authors $[5,6,8-10]$.

Due to the small physical size, the MOSFET detector can be conveniently introduced to the nasopharynx via the small nasopharyngeal applicator making it attractive for in vivo treatment verification. Before use each MOSFET detector should be calibrated individually against an ionization chamber in the water phantom. There are some reports that the radiation response of the MOSFETs may not completely linear over the whole voltage range $[15,17]$. As a result, a recalibration of the device was performed periodically for every $5-\mathrm{V}$ increment in our experiments.

The MOSFET detectors are known to have photon energy dependence in the low energy range of the ${ }^{192}$ Iridium spectrum $[15,23]$. The previously published data showed that a correction factor of about 1.27 times greater than the $5-\mathrm{cm}$ value was required in absolute depth dose determination at $1-\mathrm{cm}$ distance from the source for a RADFET dosimeter [15]. In this updated generation products, the energy response of the MOSFET detector in the ${ }^{192}$ Iridium spectrum has been optimized by using special encapsulation techniques that avoid high $\mathrm{Z}$ materials close to the dosimetric volume. The sensitivity variation, as shown by our results, was minimized to be within $7 \%$ in 
the clinically relevant distance range of $1-5 \mathrm{~cm}$. This will help improve the dosimetric accuracy of in vivo measurement for HDR brachytherapy.

The reproducibility of MOSFET measurements in the manual mode was found to be $1.4 \%$ at the threshold voltage shift of about $100 \mathrm{mV}$. This result was consistent with previous findings [24]. Due to electronic noise, the real time data acquisition mode exhibited a relatively large deviation in reproducibility tests (i.e. 1.9\%). Considering normal HDR treatments usually deliver a dose that can cause a threshold voltage change higher than $100 \mathrm{mV}$ to the measurement point, it is practically within the measurement uncertainty.

Among kinds of QA problems for HDR brachytherapy, the accurate placement of the applicator is crucial for reliable dose distribution and treatment outcome. Kremer et al developed a computer-assisted device for three-dimensional placement of afterloading probes based on CT scans [25]. With this device, target points have been reached with a mean accuracy of $0.6 \mathrm{~mm}$ (maximum deviation was $2.6 \mathrm{~mm}$ ). At our institution, we treated NPC patients using a customized nasopharyngeal applicator on an individual basis as introduced by $\mathrm{Ng}$ et al [3]. By improving the applicator design with the indicated lengths and angles as well as immobilizing the patient head and the applicator with a face mask, the inaccuracy of applicator positioning can be minimized to the same level as the complex computer-assisted system. The preliminary results from a total of 70 in vivo measurements with real time MOSFET dosimetry show good agreement between theory and experiment. The observed mean 
differences between the actually delivered and the planned dose within a single treatment fraction were $-0.1 \pm 3.8 \%$ and $-0.1 \pm 3.7 \%$, respectively, for right and left side assessments. More research is warranted.

Unlike standard fractionated radiation therapy, the HDR treatment delivers a very high dose over a few fractions. In such condition, real time dosimetry is most valuable in detecting a dose error at the onset of treatment and providing the capability of immediate corrections. According to the previously published document [5], more than $20 \%$ total dose deviation from prescription was taken as our action level to avoid "significant errors". However, for a treatment with measurable 10\% 20\% dose difference, a retrospective QA review was also required. By means of online comparison between measured doses and calculated values, the validity of HDR treatment was ensured. The measured average dose over all fractions of a patient differed from the calculated value all within $-5 \%$ to $7 \%$ in our experiment.

The presented study has major focus on the development of a simple point dose method for in vivo monitoring and verification of HDR treatments. Hence only a single MOSFET detector was evaluated in our phantom measurements and in vivo applications. Price and his coworkers have recently presented a new linear MOSFET-array dosimeter configuration that can provide a one-dimensional dose profile across the target volume [26]. As the detector array may better serve the purpose of in vivo patient dosimetry, it is now under research by ours and other groups. In future developments the external dimensions of the array dosimeter will be 
significantly reduced so as to permit its use in brachytherapy applicators. Anyhow, we feel that even with currently designed MOSFETs, the proposed method is capable of detecting errors of major clinical significance.

\section{Conclusion}

In vivo measurement using the real-time MOSFET dosimetry system provides a means of overall dosimetry check for the HDR treatment. With detectors placed within the patients' nasopharynx, it is possible to evaluate and document, in real time, the actual dose to the tumor received by the patient during a treatment fraction. Through an online comparison between the measured dose and the calculated value, the dosimetry system offers another line of security to detect and prevent large errors. The method we presented here is universal and can be applied in any other cases of clinical interest.

\section{Acknowledgement}

This research was supported by National Natural Science Foundation of China Grant No.30900324.

\section{Conflict of Interest}

We declare that we have no financial and personal relationships with other people or organizations that can inappropriately influence our work.

\section{References}


1 Levendag PC, Schmitz PI, Jansen PP, Eijkenboom WM, Visser AG, Kolkman-Deurloo IK, Sipkema D, Visch LL, Senan S. Fractionated high-dose-rate brachytherapy in primary carcinoma of the nasopharynx. J Clin Oncol 16(6): 2213-2220 (1998).

2 Levendag PC, Lagerwaard FJ, de Pan C, Noever I, van Nimwegen A, Wijers O, Nowak PJ. High-dose, high-precision treatment options for boosting cancer of the nasopharynx. Radiother Oncol 63(1): 67-74 (2002).

3 Ng T, Richards G.M, Emery RS, Ho G., Yung R, Cheng A, Berson AM. Customized conformal high-dose-rate brachytherapy boost for limited-volume nasopharyngeal carcinoma. Int J Radiat Oncol Biol Phys 61(3): 754-761 (2005).

4 Kubo HD, Glasgow GP, Pethel TD, Thomadsen BR, Williamson JF. High dose-rate brachytherapy treatment delivery: Report of the AAPM Radiation Therapy Committee Task Group No. 59. Med Phys 25(4): 375-403, 1998.

5 Alecu R, Feldmeier JJ, Court WS, Alecu M, Orton CG. A method to avoid misadministrations in high dose rate brachytherapy. Med Phys 24(2): 259-261 (1997).

6 Duan J, Macey DJ, Pareek PN, Brezovich IA. Real-time monitoring and verification of in vivo high dose rate brachytherapy using a pinhole camera. Med Phys 28(2): 167-173, 2001.

7 Wan G, Wei Z, Gardi L, Downey DB, Fenster A. Brachytherapy needle deflection evaluation and correction. Med Phys 32(4): 902-909 (2005).

8 Alecu R, Alecu M. In-vivo rectal dose measurements with diodes to avoid misadministrations during intracavitary high dose rate brachytherapy for carcinoma of the cervix. Med Phys 26(5): 768-770 (1999).

9 Brezovich IA, Duan J, Pareek PN, Fiveash J, Ezekiel M. In vivo urethral dose measurements: a method to verify high dose rate prostate treatments. Med Phys 27(10): 2297-2301 (2000).

10 Anagnostopoulos G, Baltas D, Geretschlaeger A, Martin T, Papagiannis P, Tselis $\mathrm{N}$, Zamboglou N. In vivo thermoluminescence dosimetry dose verification of transperineal ${ }^{192}$ Ir high-dose-rate brachytherapy using CT-based planning for the treatment of prostate cancer. Int J Radiat Oncol Biol Phys 57(4): 1183-1191, 2003.

11 Izewska J, Georg D, Bera P, Thwaites D, Arib M, Saravi M, Sergieva K, Li K, Yip FG, Mahant AK, Bulski W. A methodology for TLD postal dosimetry audit of high-energy radiotherapy photon beams in non-reference conditions. Radiother Oncol 84(1): 67-74 (2007). 
12 Ho AK, Gibbs IC, Chang SD, Main B, Adler JR. The Use of TLD and Gafchromic Film to Assure Submillimeter Accuracy for Image-Guided Radiosurgery. Med Dosim 33(1): 36-41 (2008).

13 Zhu XR. Entrance dose measurements for in-vivo diode dosimetry: Comparison of correction factors for two types of commercial silicon diode detectors. J Appl Clin Med Phys 1(3): 100-107 (2000).

14 Saini AS, Zhu TC. Dose rate and SDD dependence of commercially available diode detectors. Med Phys 31(4):914-924 (2004).

15 Zilio VO, Joneja OP, Popowski Y, Rosenfeld A, Chawla R. Absolute depth-dose-rate measurements for an ${ }^{192}$ Ir HDR brachytherapy source in water using MOSFET detectors. Med Phys 33(6): 1532-1539 (2006).

16 Qi ZY, Deng XW, Huang SM, Lu J, Lerch M, Cutajar D, Rosenfeld A. Verification of the plan dosimetry for high dose rate brachytherapy using metal-oxide-semiconductor field effect transistor detectors. Med Phys 34(6): 2007-2013 (2007).

17 Fagerstrom JM, Micka JA, DeWerd LA. Response of an implantable MOSFET dosimeter to ${ }^{192}$ Ir HDR radiation. Med Phys 35(12): 5729-5737 (2008).

18 Gladstone DJ, Chin LM. Automated data collection and analysis system for MOSFET radiation detectors. Med Phys 18(3): 542-548 (1991).

19 Kaplan GI, Rosenfeld AB, Allen BJ, et al. Improved spatial resolution by MOSFET dosimetry of an x-ray microbeam. Med Phys 27(1): 239-244 (2000).

20 Rosenfeld AB, Lerch MLF, Kron T, Brauer-Krisch E, Bravin A, Holmes-Siedle A, Allen BJ. Feasibility study of online high-spatial-resolution MOSFET dosimetry in static and pulsed X-ray radiation fields. IEEE Trans Nuc Sci 48(6): 2061-2068 (2001).

21 Kwan IS, Wilkinson D, Cutajar D, Lerch M, Rosenfeld A, Howie A, Bucci J, Chin Y, Perevertaylo VL. The effect of rectal heterogeneity on wall dose in high dose rate brachytherapy. Med Phys 36(1): 224-232 (2009).

22 Reynaert N, Verhaegen F, Thierens H. In water calibration of PDR ${ }^{192} \mathrm{Ir}$ brachytherapy sources with an NE2571 ionization chamber. Phys Med Biol 43(8): 2095-2107(1998).

23 Kron T, Duggan L, Smith T, Rosenfeld A, Butson M, Kaplan G, Howlett S, Hyodo K. Dose response of various radiation detectors to synchrotron radiation. Phys Med Biol 43(11): 3235-3259 (1998).

24 Cheung T, Yu PKN, Butson MJ. Low-dose measurement with a MOSFET in 
high-energy radiotherapy applications. Radiat Meas 39(1): 91-94 (2005).

25 Kremer B, Klimek L, Andreopoulos D, Mösges R. A new method for the placement of brachytherapy probes in paranasal sinus and nasopharynx neoplasms. Int J Radiat Oncol Biol Phys 43(5): 995-1000, 1999.

26 Price RA, Benson C, Joyce MJ, Rodgers K. Development of a RadFET linear array for intracavitary in vivo dosimetry during external beam radiotherapy and brachytherapy. IEEE Trans Nucl Sci 51(4): 1420-1426 (2004). 
Table 1 The MOSFET readout reproducibility in different data acquisition modes at given dose levels.

\begin{tabular}{ccc}
\hline \multirow{2}{*}{$\begin{array}{c}\text { Dose level } \\
(\mathrm{cGy})\end{array}$} & \multicolumn{2}{c}{ MOSFET readout reproducibility } \\
\cline { 2 - 3 } & Manual mode & Real time mode \\
\hline 20 & $3.5 \%$ & $4.8 \%$ \\
30 & $2.4 \%$ & $3.2 \%$ \\
50 & $1.4 \%$ & $1.9 \%$ \\
\hline
\end{tabular}

\section{Lists of Figures}


Figure 1 A custom-made nasopharyngeal applicator. The imprinted lengths and angles on the applicator are used for daily applicator positioning.

Figure 2 Comparison of energy-dependent correction factors between the new MOSkin $^{\mathrm{TM}}$ and a previously used RADFET dosimeter. The RADFET data were derived from the published data [8], which were re-normalized to the 5-cm value.

Figure 3 An example of real time dose variations between in vivo measurements and calculated accumulated doses for a given treatment fraction. The MOSFET measurements coincided with the calculated values throughout the treatment delivery.

Figure 4 The frequencies of measurements with discrepancies in percentage between measured and calculated values for right and left side measurements, respectively.

Figure 5 The discrepancies between measured and planned doses averaged over all fractions of a patient. The error bar represents one standard deviation. 